\title{
Tiempo en pantalla (televisión, computadora, celular, tabletas) en las relaciones interpersonales entre niños de 8 a 12 años
}

\author{
Screen time (television, computer, cell phone, tablets) in relationships \\ interpersonal between children 8 to 12 years
}

Tempo de tela (televisão, computador, telefone celular, tablets) nos relacionamentos interpessoal entre crianças de 8 a 12 anos

\section{ARTÍCULO ORIGINAL}

Kerly Angela Álvarez Cadena

kalvarezc@unemi.edu.ec

ORCID: 0000-0003-0971-1178

Diana Elizabeth Pilamunga Asacata

dpilamungaa@unemi.edu.ec

ORCID: 0000-0002-1921-9024

\author{
Karla Gabriela Mora Alvarado \\ kmoraa@unemi.edu.ec \\ ORCID: 0000-0003-1265-0716
}
Miossotty Katherine Naranjo Kean Chong
mnaranjok2@unemi.edu.ec
ORCID: 0000-0001-6599-303X

Universidad Estatal de Milagro, Ecuador

Recibido abril 2020 | Revisado mayo 2020 | Publicado en julio 2020

\section{RESUMEN}

El relacionamiento frecuente de la tecnología en los niños es un tema que dentro del contexto actual se considera un problema, debido a un notable decrecimiento en las relaciones interpersonales, este efecto se da por el tiempo que dedican a los medios tecnológicos, dejando en segundo plano el factor de relaciones interpersonales. El estudio tuvo como objeto detectar la influencia del tiempo de pantalla en las relaciones interpersonales, la metodología empleada fue de tipo cuantitativa, descriptiva y correlacional. Se seleccionó una muestra representativa conformada por 100 adolescentes de instituciones educativas fiscales de la Ciudad de Milagro. Los instrumentos utilizados fueron el Cuestionario de Estilo Interpersonal para Adolescentes (CEI-A). Los resultados indicaron que existe correlación débil y significativa entre los factores asociados a los estilos de relación interpersonal y el tiempo que pasan frente a las pantallas [ $\left.\left(\mathrm{r}=052^{*}\right) ;\left(\mathrm{p}=179^{*}\right) ;\left(\mathrm{p}=431^{* *}\right)\right]$, con lo cual se concluyó que existen efectos negativos en el desarrollo de habilidades sociales de los niños.

Palabras clave: Tecnología; tiempo; relaciones interpersonales; desarrollo, habilidades

\section{ABSTRACT}

The frequent relationship of technology in children is an issue that in the current context is considered a problem, due to a notable decrease in interpersonal relationships, this effect is given by the time they spend on technological means, leaving the factor of interpersonal relationships in the background. The aim of the study was to detect the influence of screen time on interpersonal relationships. The methodology used was quantitative, descriptive and correlational. A representative sample of 100 adolescents from public educational institutions in the City of Miracles was selected. The instruments used were the Interpersonal Style Questionnaire for Adolescents (CEI-A), its factors are: assertiveness and aggressiveness, the total reliability of the scale is .67, and the Zero to Eight survey to measure time on screen, whose factors were the use of television and electronic media. The results indicated that there is a weak and significant correlation between the factors associated with interpersonal relationship styles and the time spent in front of the screens $\left[\left(\mathrm{r}=052^{*}\right) ;\left(\mathrm{p}=179^{*}\right) ;\left(\mathrm{p}=431^{* *}\right)\right]$, concluding that there are negative effects on the development of social skills in children.

Key words: Technology; time; interpersonal relationships; development, skills 


\section{RESUMO}

A utilização frequente da tecnologia pelas crianças é uma questão que no contexto atual é considerada um problema, devido a uma diminuição notável nas relações interpessoais, este efeito é dado pelo tempo que gastam em meios tecnológicos, deixando o fator das relações interpessoais como pano de fundo. 0 objetivo do estudo era detectar a influência do tempo na tela nas relações interpessoais. A metodologia utilizada era quantitativa, descritiva e correlativa. Foi selecionada uma amostra representativa de 100 adolescentes de instituições educativas públicas da Cidade dos Milagres. Os instrumentos utilizados foram o Questionário de Estilo Interpessoal para Adolescentes (CEI-A), os seus fatores são: assertividade e agressividade, a fiabilidade total da escala é de.67, e o inquérito Zero a Oito para medir o tempo na tela, cujos fatores foram a utilização da televisão e dos meios electrónicos. Os resultados indicaram que existe uma fraca e significativa correlação entre os fatores associados aos estilos de relacionamento interpessoal e o tempo passado em frente nas telas [( $\left.\left.\mathrm{r}=052^{*}\right) ;\left(\mathrm{p}=179^{*}\right) ;\left(\mathrm{p}=431^{* *}\right)\right]$, concluindo que existem efeitos negativos no desenvolvimento de competências sociais nas crianças.

Palavras-chave: Tecnologia; tempo; relações interpessoais; desenvolvimento, competências

\section{INTRODUCCIÓN}

En la actualidad el uso de la tecnología se ha vuelto una nueva tendencia, cada día existen nuevas formas y plataformas para interacción entre individuos, así como sus múltiples aplicaciones. Sin embargo, el uso desmedido o inadecuado puede ocasionar efectos adversos sobre la población, dado que la falta de control es una debilidad que genera una dependencia entre otros efectos.

Con la aparición de las primeras pantallas como difusores de la comunicación, se ha expandido la preocupación por el impacto que puede provocar el uso de estas en los niños. Dicha preocupación se ha acrecentado en la actualidad por el uso generalizado de las nuevas tecnologías según Paniagua Repetto, (2013), no solo por las constantes amenazas a las que se exponen debido al cyber acoso, cyberbulling o el groming, la mayor preocupación radica en los espacios que los medios tecnológicos se arraigan desplazando otras actividades decisivas para el desarrollo (Pérez Porto y Merino, 2016).

El tema de la exagerada manipulación de medios tecnológicos y sobre todo el tiempo que dedican los estudiantes a aquello, se ha vuelto incontrolable desde los hogares; también se ha podido evidenciar que cada vez los infantes tienen acceso mayoritario a este tipo de dispositivos, lo que representa un serio problema, dejando un efecto significativo en el desarrollo de sus habilidades sociales para su entorno. Realizar un diagnóstico social sobre este tipo de fenómenos contribuye a la comunidad a dotarlos de estrategias de control y sobre todo una educación sobre la utilidad y consecuencias de su uso exagerado y el tiempo en pantalla.

En las instituciones educativas primarias, del cantón Milagro, los departamentos de consejería estudiantil han reportado una considerable cifra de estudiantes que tienen problemas en su desarrollo social, el comportamiento es agresivo y evasivo, y además mencionan que se ocupan mucho con sus dispositivos móviles, por lo que también descuidan sus responsabilidades académicas. Este malestar deja efectos negativos que afectan de forma directa el círculo social de los estudiantes, es probable que dentro de los hogares no exista el debido cuidado y control sobre sus actividades, dado su comportamiento dentro de los planteles. Este tipo de situaciones se las puede mediar una vez determinado el problema, el trabajo mancomunado entre docentes y padres de familia sería de gran ayuda para poder mejorar las condiciones de los niños.

En este estudio se analizó la relación entre el tiempo que los estudiantes pasan en pantalla, es decir el uso frecuente de medios tecnológicos, y las relaciones interpersonales. El 
ser humano por naturaleza es un agente social que requiere comunicación para desarrollarse y poder responder a demandas externas. Esta investigación contribuye al contexto educativo en gran medida, debido que, con los resultados obtenidos, se pueden formular pautas para mejorar las debilidades y efectos que conlleva el exagerado uso de tecnología

\section{MÉTODO}

Fue un estudio de tipo no experimental de corte transversal y de enfoque descriptivo y correlacional, se utilizó un cuestionario relacionado a la exposición del tiempo de pantalla, y el otro cuestionario mide relaciones interpersonales. Se aplicó la encuesta a 100 estudiantes correspondientes a instituciones educativas de formación básica, las edades oscilan entre 8 y 12 años con una mayor frecuencia en niños de 12.

Con relación a los instrumentos se ha elegido al cuestionario de Estilo Interpersonal para Adolescentes Bo Wen (1986). Aplicable en edades entre 12 y 20 años. La fiabilidad total de la escala es de.67. la fiabilidad de los factores es de .70 y .68 respectivamente, se compone de 11 ítems que permitió la evaluación del estilo de comunicación interpersonal desglosados en la siguiente manera: Asertividad (Ítems 2,4,6,8,9,11), Agresividad (Ítems 1,3,5,7,10). Los ítems se presentaron como opciones de respuestas: Nunca, a veces, con frecuencia, siempre, las puntuaciones.

Mientras que para medir el tiempo en pantalla, se utilizó el Cuestionario Zero to Eight cuyos autores son Rideout, Saphir, y Common (2011) desarrollados en temáticas de tecnología de la información y la comunicación con temáticas en dispositivos móviles, tabletas; este cuestionario tiene como objetivo entender los patrones de uso de las TIC, estudiando el tiempo dedicado y la frecuencia de uso de la tv, el ordenador, el teléfono móvil y otros dispositivos digitales, también las diferencias en el uso según el sexo, la raza o estatus socioeconómico, esta prueba está compuesta por 26 ítems compuesto por preguntas abiertas y otras con alternativas como por ejemplo sí o no, los padres son los que responden las preguntas relacionadas desde la edad del niño hasta el tiempo de la exposición del infante. Para analizar los datos fue utilizado el programa estadístico SPSS-V25, para lo cual se procedió a ingresar los datos para efectuar el procesamiento de los mismos, se inició con un análisis de frecuencia desde los factores edad y sexo, luego se realizó la correlación bivariada de Pearson y estadísticas descriptiva de toda la base.

\section{RESULTADOS}

En la tabla 1 se muestran los estadísticos descriptivos de las variables de edad, este proceso como resultado de los instrumentos aplicados, donde se evidenció que la media en edad es de 11,17, la mediana es 12 al igual que la moda, lo que establece que los participantes oscilan entre 11 y 12 años de edad, manteniendo una desviación típica de 2,146 de margen. Esto quiere decir que no hay mucha distancia entre las edades de los niños, es decir, las diferencias en meses y además que la mayoría de participantes son de 12 años. 
Tabla 1. Análisis descriptivo de variable edad

\begin{tabular}{llc} 
Edad & Válido & \\
N & Perdidos & 100 \\
Media & & 0 \\
Mediana & 11,17 \\
Moda & 12,00 \\
Desv. Desviación & 12 \\
Varianza & 2,146 \\
\hline
\end{tabular}

Se puede observar en la figura 1 que se utilizó para analizar la frecuencia de edades de participantes, representado en un histograma, donde se visualizó que la curva de normalidad esta inclinada hacia la derecha, esto indicó que las edades más constantes de evaluados estuvieron entre 11 y 12 años.

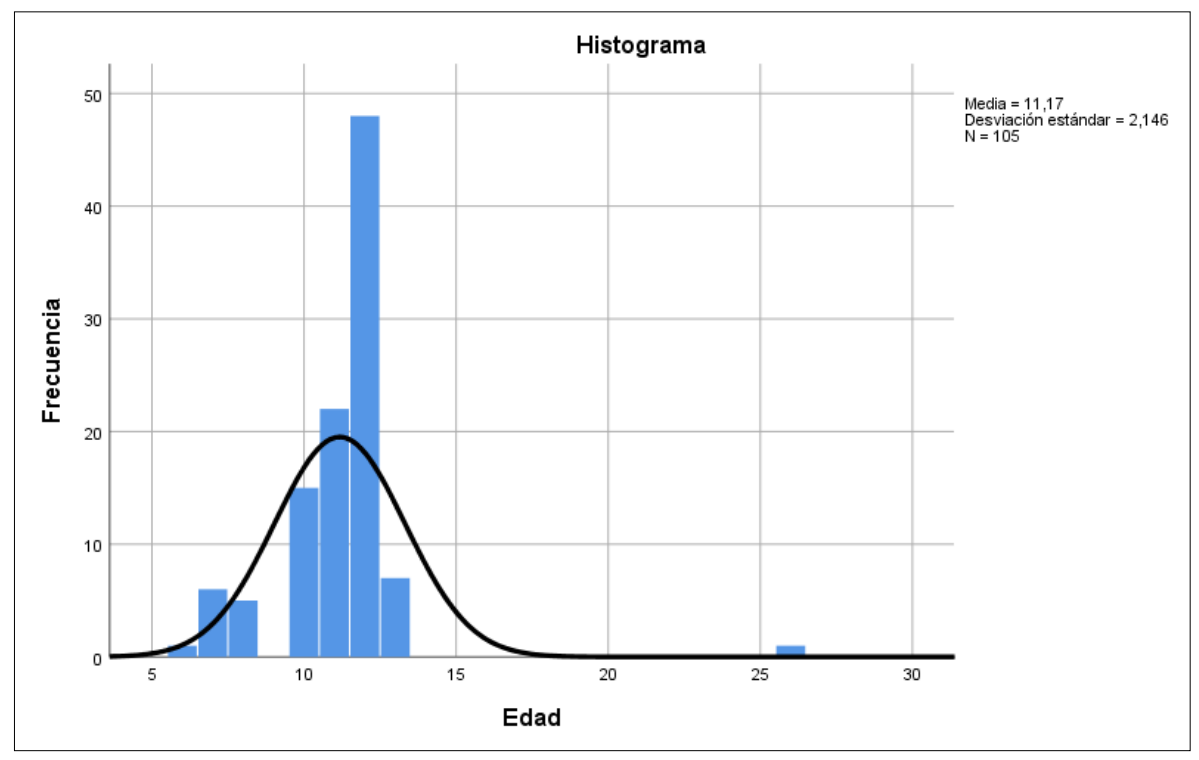

Figura 1. Análisis de frecuencia de variable edad

En la figura 2 se puede observar la frecuencia de la variable sexo entre los participantes evaluados, y en lo que respecta la curva de normalidad, se evidenció una posición media, con lo cual se mencionó que el sexo de los niños de la muestra estas distribuidos por igual, esto quiere decir que, existen cantidades similares tanto de varones como mujeres. 


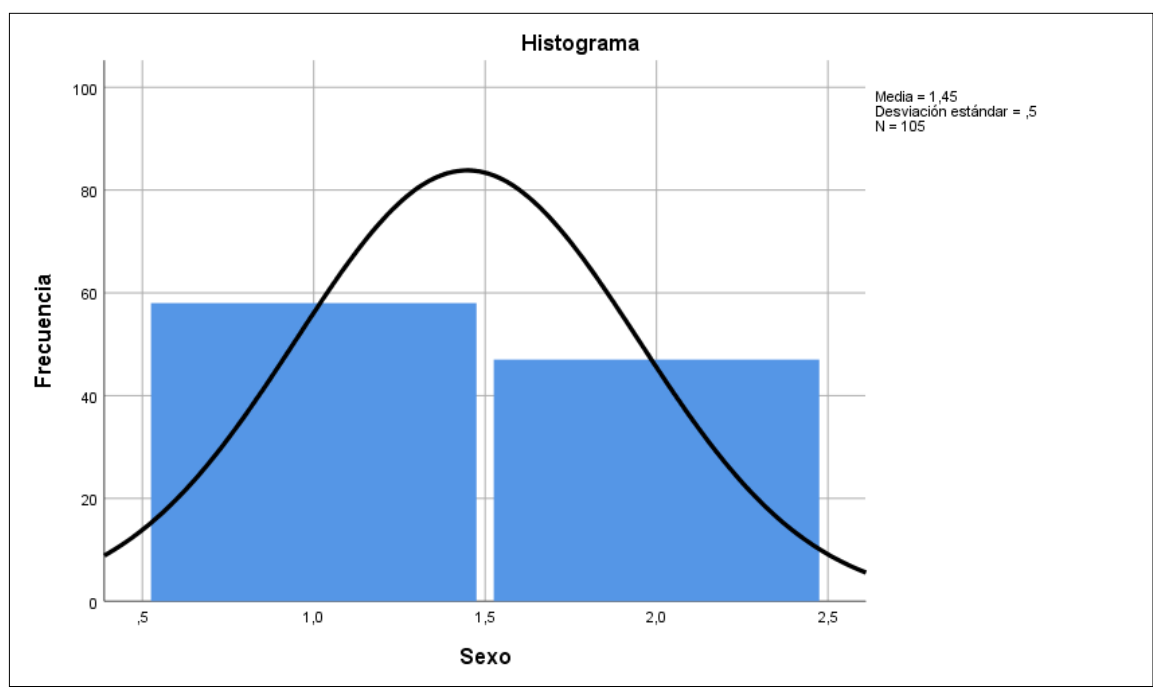

Figura 2. Análisis de frecuencia de variable sexo

En la tabla 2 se puede evidenciar mediante el método de correlación de Pearson la relación entre los factores del cuestionario de relaciones interpersonales y el cuestionario Zero to Eight, donde se demostró que existe una correlación significante débil entre asertividad y el uso de televisión $\left(\mathrm{r}=052^{*}\right)$; sin correlación entre asertividad y uso de dispositivos móviles. Por otro lado, el estilo agresivo se correlaciona débilmente con el uso de la televisión ( $\mathrm{p}=179^{*}$ ) y una correlación altamente significativa con el uso de dispositivos móviles ( $\left.\mathrm{p}=431^{* *}\right)$. Estos resultados quieren decir, que existe agresividad en los niños que frecuentan el uso de la televisión y aún más en lo que refiere a uso de dispositivos móviles, lo que genera un efecto desfavorable para su comportamiento, así como no tener habilidades sociales adecuadas para su contexto.

Tabla 2. Análisis correlacional de factores

\section{Correlaciones}

\begin{tabular}{llcr} 
Asertividad & Correlación de Pearson &, $052^{*}$ &, 082 \\
& Sig. (bilateral) &, 005 &, 004 \\
\multirow{2}{*}{ Agresividad } & $\mathrm{N}$ & 105 & 105 \\
& Correlación de Pearson &, $179^{*}$ &, $431^{* *}$ \\
\multirow{2}{*}{ Uso de televisión } & Sig. (bilateral) &, 004 &, 001 \\
& $\mathrm{~N}$ & 105 & 105 \\
& Correlación de Pearson &, $052^{*}$ &, 082 \\
\multirow{2}{*}{ Uso de dispositivos móviles } & Correlación de Pearson &, 005 & 104 \\
& Sig. (bilateral) & 105 &, $431^{* *}$ \\
& $\mathrm{~N}$ &, $179 *$ &, 001 \\
\hline
\end{tabular}

*. La correlación es significativa en el nivel 0,05 (bilateral)

**. La correlación es significativa en el nivel 0,01 (bilateral). 


\section{Discusión}

En otros estudios se ha podido determinar que el 29\% de niños menores de 1 año de edad observan la TV y videos alrededor de 90 minutos al día. El 64\% de niños entre 12 y 24 meses no pierden de vista la TV y vídeos durante más o menos 2 horas diarias, cabe recalcar que el consumo visual de los dispositivos electrónicos o la TV aumenta con la edad del usuario (Linn, Wolfsheimer Almon, y Levin, 2012). Esta actividad que por lo regular sucede en niños se puede incrementar, por lo cual su relacionamiento personal se puede reducir en la medida que no es regulado los espacios de interacción con otras personas y con sus medios electrónicos.

Según Pempek, Kirkorian, y Anderson,( 2014) la permanencia de la televisión como ruido de fondo afecta en gran medida el desarrollo del lenguaje de los niños. En este mismo estudio se constató que el $74 \%$ de los niños de la muestra, menores de 5 años, empezaron a ver televisión antes de los dos años de edad lo cual está contraindicado por la Academia Americana de Pediatría. Para la Canadian Paediatric Society (2017) la alta exposición a TV de fondo afecta negativamente los procesos cognitivos de la atención, el desarrollo de la función ejecutiva, así como el uso y la adquisición del lenguaje en niños menores de 5 años.

Por otro lado, algunos estudios han demostrado que el exceso de tiempo de pantalla está ligado con el desarrollo de trastornos de aprendizaje, déficit de atención y trastornos del sueño (Linn, Wolfsheimer Almon, y Levin, 2012), en comparación con los resultados encontrados, se puede precisar que el estilo agresivo puede desencadenar a largo plazo en un posible trastorno de la conducta.

De acuerdo a Vera Vila, (2013) cualquier cambio en la tecnología de comunicación humana tiene efectos sobre el contenido de los mensajes, los modos y medios de pensar, reflexionar, expresarse, argumentar o de recordar; en definitiva, sobre las propias estructuras y funciones cognitivas, es así que el medio cada vez más tecnificado plantea diversos paradigmas, pros y contras que no clarifican sino más bien postergan las conclusiones.

Coincidiendo con Lillard, Li, y Boguszewski, (2015) discurren que ciertos programas de televisión perjudican la función ejecutiva subsiguiente, ya que ejecutar tareas concernientes a la función ejecutiva y ver la televisión manejan los mismos recursos de procesamiento de la información. Consideran además, que tales programas de televisión pueden generar determinados tipos de conductas en los niños, por lo cual es recomendable monitorear el tipo de programación que visualizan.

Uno de los riesgos provocados fundamentalmente por un uso inadecuado de este medio, los niños pueden no discriminar la realidad de la ficción y todos son vulnerables frente a los mensajes recibidos, que pueden influir negativamente en sus comportamientos, puede dificultar la comunicación familiar y restar tiempo para el juego, la lectura, el deporte o el paseo, la visualización de la violencia, muy frecuente en la televisión puede aparecer como un hecho aceptable y cotidiano para dirimir disputas o ser una conducta a imitar, el exceso de horas frente a esta pantalla ha sido relacionado con un mal rendimiento académico $\mathrm{y}$ con conductas agresivas $\mathrm{y}$ violentas, la exposición reiterada a la violencia es un riesgo de conductas agresivas a corto y largo plazo, pero no todos los niños $\mathrm{y}$ adolescentes expuestos a ella las padecerán; por lo que, hay que considerar otros factores ajenos a esta tecnología, personales o sociales, como inductores de estos comportamientos (Paniagua, 2013).

Así se puede decir que el atractivo de las tecnologías para los más pequeños, unido a su 
fácil acceso puede provocar que se vean involucrados por ellas. Hecho que en la actualidad preocupa a una gran cantidad de expertos por las consecuencias negativas que esto pueda acarrear en su desarrollo. Un estudio realizado por Peirce, (2013), titulado "Digital game-based learning for early childhood", afirma que, aunque aumentando el uso de juegos educativos informatizados, gracias a la proliferación de las tabletas, los beneficios constatados para el desarrollo infantil en edades de 3 a 6 años son más bien escasos.

Con respecto al comportamiento en niños que usan teléfonos móviles para jugar a tan temprana edad pueden verse afectados con alteraciones de su desarrollo con el entorno y daños neurológicos (De la Rosa, 2014),se hace necesario resaltar lo expuesto por Aztecas Noticias, (2015) cuando consideran que el uso del celular se convierte en un hábito perjudicial por el tiempo en que la persona permanece en el mismo, con todas las implicaciones que esto conlleva al exponerse a estímulos agradables y desagradables.

Llegando a este punto Acero (2017) manifiesta que el uso inmoderado del teléfono celular o móvil es una clase de adicción a la tecnología que puede contribuir a desarrollar actitudes de dispersión, ansiedad e intolerancia e incluso actitudes delictivas para financiar la adicción. Son más vulnerables las personas jóvenes, que desean tener siempre la última versión tecnológica, con lo que sienten que mejora su status y su autoestima, y no pueden tener ratos de silencio y de soledad, que permiten pensar, hacer tareas cotidianas, dedicar un tiempo a la lectura o ser más uno mismo (Rodríguez Cano, y otros, 2012). La adicción al teléfono esconde tras ella problemas tales como la inseguridad, baja autoestima, necesidad de sentir experiencias intensas, soledad, pobres habilidades sociales o la necesidad de mantenerse continuamente en contacto con otras personas.

Estos dispositivos cumplen las expectativas de los adolescentes en cuanto a diseño y prestaciones (posibilidades para enviar mensajes, acceso a redes sociales, capacidades interactivas, conectividad, entre otros), por lo que han tenido buena acogida en este sector poblacional (Christakis, Ramirez, y Ramirez, 2012). Los niños también están más dispuestos a dedicar tiempo y esfuerzo en aprender a utilizar las numerosas funciones que les brindan estos aparatos, y suelen adquirir las destrezas implicadas en dichas aplicaciones con mayor rapidez que los adultos (Chóliz, Villanueva, y Chóliz, 2009).

Siguiendo a Delgado, Oliva, y SánchezQueija, (2011), durante la segunda década de la vida se produce una adquisición de competencias sociales que facilitan la vinculación con el grupo y la consolidación de las relaciones de amistad. Este es el motivo por el cual las redes han triunfado entre los adolescentes, ya que satisfacen una importante necesidad natural de este colectivo: la socialización. Estas aplicaciones actúan como instrumentos de interacción ente pares, reforzando su sentimiento de pertenencia a un grupo.

Los niños se relacionan de forma diferente a la que estamos acostumbrados los adultos y tienen más canales para hacerlo. Pueden establecer relaciones que comienzan online $\mathrm{y}$ llevarlas al mundo físico, $\mathrm{y}$, al contrario. Emergen nuevas formas de relación para las que hay que desarrollar nuevas habilidades. Este proceso requiere aprendizaje $\mathrm{y}$ lógicamente, también está sujeto a un mal uso. Se ha especulado mucho sobre las bondades o los perjuicios que pueden ocasionar las redes sociales y si las relaciones en la red son más superficiales que en la vida real (Ruiz, Sánchez, y Trujillo, 2015). Por ejemplo, para Herrero, Meneses, Valiente, y Rodríguez, (2004) la participación en internet podría disfrutar de un 
estatus similar a la intervención presencial en su relación positiva con el bienestar psicológico. Para Rosen (2011), el uso excesivo diario de los medios de comunicación y la tecnología tiene un efecto negativo en la salud de todos los niños, preadolescentes y adolescentes, haciéndolos más propensos a la ansiedad, la depresión.

Un estudio reciente sugiere que las personas son propensas a ser sinceras cuando describen sus emociones a través de mensajes de texto, dado que responden sin la presión del tiempo y las respuestas son más exactas. Además, las personas son más proclives a revelar información delicada a través de este medio puesto que no existe el contacto visual (Conrad y Schober, 2012).

\section{CONCLUSIONES}

Se ha podido dejar en evidencia mediante los resultados obtenidos que la frecuencia de uso de todo tipo de dispositivo visual y auditivo en los niños posee sus grados de desventaja en la medida que se use, en el caso de no existir un control en el hogar, el acelerado y concentrado manejo de celulares, tabletas, o pasar largas horas en la televisión minimiza su dinámica relacional con su contexto.

En cuanto a resultados de estilos de relacionamiento, se ha podido observar que los participantes llevan una tendencia moderadamente abierta a desarrollar problemas de comportamiento, lo cual puede deberse al efecto del tipo de contenido que manejan en los dispositivos móviles o de los programas que percibe en la televisión.

El estilo asertivo guarda escasa relación con los factores vinculados al uso de medios tecnológicos, esto quiere decir que no desarrollan debidamente habilidades sociales propias y acordes a su edad, dado la mala administración de sus tiempos en sus actividades de recreación.

Mediante el análisis sobre el impacto del tiempo de pantalla en las relaciones interpersonales de los niños se puede afirmar que existe una relación directa, siendo el impacto de pantalla una de las razones más importantes para los problemas en las relaciones interpersonales de los adolescentes.

Se puede manejar este tipo de problemáticas manteniendo un dialogo con los padres, para lo cual es pertinente ejecutar un plan de intervención basado en aspectos relacionados a la comunicación y al tiempo que deben compartir con sus hijos, dado que muchas veces la ausencia de sus figuras paternas inclina a los infantes a buscar refugio en elementos que no son saludables cuando sus usos son exagerados.

Por otra parte, es necesario también vincular a los niños en actividades de recreación, entre ellos juegos de futbol, disciplinas deportivas de entrenamiento, o juegos tradicionales acordes a su edad, sobre todo en aquellas actividades donde les permiten socializar para poder desarrollar y fortalecer las competencias sociales.

\section{REFERENCIAS}

Acero, L. (octubre de 2017). Análisis del uso del celular y su influencia negativa en el rendimiento académico de los estudiantes de los terceros años de bachillerato de la unidad educativa colegio nacional galápagos

Canadian Paediatric Society. (2017). Screen time and young children: Promoting health and development in a digital world. Paediatrics y Child Health, 461-467

Chóliz, Villanueva, y Chóliz. (2009). Ellas, ellos y su móvil. Uso, abuso (iy dependencia?) del teléfono móvil en la adolescencia. Revista Española de Drogodependencias, 34 (1), 74-88

Christakis, D., Ramirez, J. S., y Ramirez, J. M. (2012). Overstimulation of newborn mice leads to behavioral differences and deficits in cognitive performance. Scientific reports, 3

Conrad, y Schober. (2012). Texting ups truthfulness, new iPhone study suggests. 
http://home.isr.umich.edu/releases/text ing-ups-truthfulness-new-iphone-studysuggests /

De la Rosa, R. (2014). La enfermedad silenciada. La enfermedad silenciada. . Madrid : Integralia la casa natural

Delgado, Oliva, y Sánchez-Queija. (2011). Apego a los iguales durante la adolescencia y la adultez emergente. Anales de Psicología, . 27 (1), 155-163

Herrero, Meneses, Valiente, y Rodríguez. (2004). Participación social en contextos virtuales. . Psicothema, 16 (3), 456-460

Lillard, A., Li, H., y Boguszewski, K. (2015). Television and Children's Executive Function. Advances in Child Development and Behavior, 242

Linn, S., Wolfsheimer Almon, J., y Levin, D. (2012). Ante el dilema de la pantalla: Los niños pequeños, la tecnología y la educación temprana. The Campaign for a Commercial-Free Childhood and the Alliance for Childhood, 5

Paniagua Repetto, H. (2013). Impacto de las tecnologías de la información y la comunicación . PEDIATRÍA INTEGRAL, 686 $-693$

Peirce, N. (Marzo de 2013). Digital Gamebased Learning for Early Childhood

Pempek, T., Kirkorian, H., y Anderson, D. (2014). The Effects of Background
Television on the Quantity and Quality of Child-Directed Speech by Parents. Journal of Children and Media, 211-222

Pérez Porto , J., y Merino, M. (2016). Definición de tecnología de la comunicación. Obtenido de

Definicion.de:

(https://definicion.de/tecnologia-de-lacomunicacion/)

Rosen. (2011). Poke Me: How Social Networks Can Both Help and Harm Our Kids. . 119th Annual Convention of the American Psychological Association. http://www.fenichel.com/pokeme.shtml

Rodríguez Cano, R. A., Martínez Sánchez, I. C., García Rubio, M. J., Guillén Paredes, V., Valero de Vicente, M., y Díaz, S. (2012). Adicción a las nuevas tecnologías de la información y la comunicación (nTIC) y ansiedad en adolescentes. International Journal of Developmental and Educational Psychology 1 (1), 347 - 356

Ruiz-Palmero, J., Sánchez-Rodríguez, J., y Trujillo-Torres, J. M. (2015). Utilización de Internet $\mathrm{y}$ dependencia a teléfonos móviles en adolescentes. Revista Latinoamericana de Ciencias Sociales, Niñez y Juventud, 14 (2), 1357 - 1369

Vera Vila, J. (2013). Primeros Lenguajes Y últimas tecnologías para la educación. Redalic, 148 